\title{
Utilização da liraglutida na abordagem terapêutica medicamentosa da esteato- hepatite não alcoólica (EHNA)
}

\author{
Use of liraglutide in the medicinal therapeutic approach of non-alcoholic \\ steatohepatitis (NASH)
}

Uso de liraglutida em el enfoque terapéutico medicinal de la esteatohepatitis no alcohólica (EHNA)

Recebido: 30/07/2021 | Revisado: 05/08/2021 | Aceito: 10/08/2021 | Publicado: 13/08/2021

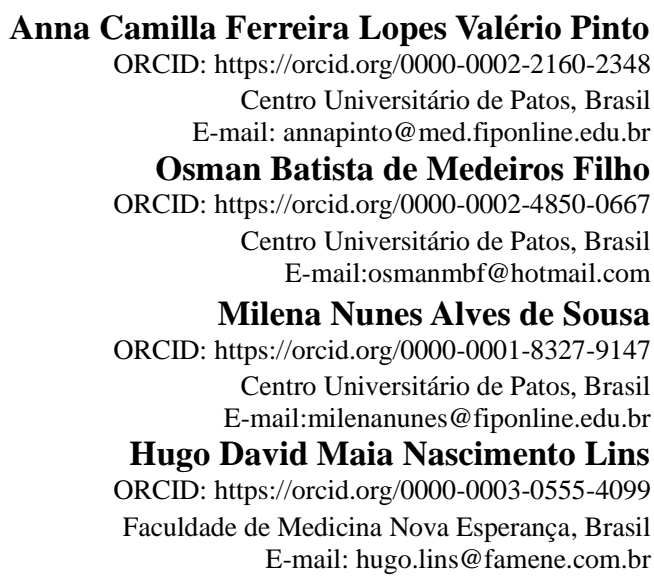

\begin{abstract}
Resumo
Avaliar os efeitos da administração de liraglutida na EHNA. Consiste em uma revisão sistemática da literatura nas principais bibliotecas virtuais: US National Library of Medicine National Institutes of Health (PubMed), Biblioteca Virtual de Saúde (BVS), Scientific Electronic Library Online (SCIELO) e Literatura Latino-americana e do Caribe em ciências da Saúde (LILACS) nos últimos cinco anos, admitindo os artigos provenientes de ensaios clínicos. A liraglutida é um análogo do hormônio peptídeo semelhante a glacagon 1 (GLP-1) que atua na supressão do apetite e da lipogênese de novo (DNL), diminuição da liberação do glucagon, aumento da liberação e sensibilização insulínica entre outros. Por esses motivos, suas frentes de combate a EHNA vão desde a diminuição e controle do perfil lipídico, até a reparação dos hepatócitos. Atualmente, com o ambiente obesogênico encontrado na sociedade, a terapia medicamentosa torna-se uma grande aliada da comunidade médica, assim como na facilitação das metas para o paciente. $\mathrm{O}$ uso da liraglutida na EHNA mostrou-se eficaz no seu desaceleramento e resolutividade, com terapias variando de 26 a 48 semanas e doses de $0,9 \mathrm{mg}$ a 3,0 $\mathrm{mg}$.
\end{abstract}

Palavras-chave: Hepatopatia gordurosa não alcoólica; Tratamento farmacológico; Liraglutida.

\begin{abstract}
To evaluate the effects of liraglutide administration on NASH. Consists of a systematic review of the literature in the main virtual libraries: US National Library of Medicine National Institutes of Health (PubMed), Biblioteca Virtual de Saúde (BVS), Scientific Electronic Library Online (SCIELO) and Latin American and Caribbean Literature in Health Sciences (LILACS) in the last five years, admitting articles from clinical trials. Liraglutide is an analogue of the glacagon 1-like peptide hormone (GLP-1) that acts in suppressing appetite and de novo lipogenesis (DNL), decreasing glucagon release, increasing insulin release and sensitization, among others. For these reasons, its fronts to combat NASH range from the reduction and control of the lipid profile, to the repair of hepatocytes. Currently, with the obesogenic environment found in society, drug therapy has become a great ally of the medical community, as well as in facilitating goals for the patient. The use of liraglutide in NASH proved to be effective in slowing and resolving it, with therapies ranging from 26 to 48 weeks and doses ranging from $0,9 \mathrm{mg}$ to 3,0 $\mathrm{mg}$.
\end{abstract}

Keywords: Non-alcoholic fatty liver disease; Drug therapy; Liraglutide. 


\section{Resumen}

Evaluar los efectos de la administración de liraglutida sobre NASH. Consiste en una revision sistemática de la literature en las principals bibliotecas virtuales: US National Library of Medicine National Institutes of Health (PubMed), Biblioteca Virtual de Saúde (BVS), Scientific Electronic Library Online (SCIELO) y Latin American and Caribbean Literature in Science (LILACS) em los últimos cinco años, admitiendo artículos de ensayos clínicos. La liraglutida es un análogo de la hormona peptídica similar al glacagón 1 (GLP-1) que actúa para suprimir el apetito y lalipogénesis de novo (DNL), diminuir la liberación de glucagón, aumentar la liberación de insulina y la sensibilización, entre otros. Por estos motivos, sus frentes para combatirla EHNA van desde la reducción y control del perfil lipídico, hasta la reparación de hepatocitos. Actualmente, conel entorno obesogénico que se vive em la sociedad, lafarmacoterapia se convierteenungran aliado de la comunidad médica, así como en facilitar metas para el paciente. El uso de liraglutidaen EHNA demostró ser eficaz en su desaceleración y resolución, con terapias que van de 26 a 48 semanas y dosis de $0,9 \mathrm{mg}$ a $3,0 \mathrm{mg}$.

Palabras clave: Enfermedad del hígado graso no alcohólico; Quimioterapia; Liraglutida.

\section{Introdução}

A esteato-hepatite não alcóolica (EHNA) faz parte do espectro da doença hepática gordurosa não alcóolica (DHGNA), sendo a principal etiologia das doenças hepáticas crônicas. Afeta $25 \%$ da população mundial e mais de $85 \%$ dos pacientes com o índice de massa corporal (IMC) acima de $40 \mathrm{~kg} / \mathrm{m}^{2}$, ou seja, obesos mórbidos (Younossiet al., 2016). É considerada, por vezes, o acometimento hepático da síndrome metabólica, a qual abrange a dislipidemia, obesidade e intolerância à insulina (Lebeaupin et al., 2018).

Além disso, a EHNA foi responsável por 7,6\% das mortes totais no mundo relacionadas a doenças hepáticas em 2015 (cerca de 28.200 óbitos), sendo esperado, no ano de 2030, que ultrapasse mais de 80 mil mortes, totalizando 10,9\%. Dessa forma, pode sobrecarregar os hospitais e os recursos da rede de saúde pública, visto que há um aumento da incidência em três vezes o número de casos em apenas 15 anos, ou seja, uma média anual de 3.340 óbitos no mundo (Estes et al., 2018). Em relação à distribuição continental, pode-se encontrar na América do Sul uma taxa de 30,5\%, seguida pela Ásia com 27,4\%, Europa com 23,7\% e África com 13,5\%. Algumas regiões e países destacam-se por razão da prevalência, como por exemplo o Oriente Médio, com 31,8\%, e os EUA, com 24,1\%. A grande mortalidade da EHNA está relacionada às complicações cardiovasculares (Araújo et al., 2018).

A EHNA tem início com o acúmulo patológico dos lipídios nos hepatócitos. Estes são responsáveis pela lipogênese, biossíntese do colesterol e metabolismo da glicose (Lebeaupin et al., 2018; Wang \& Kaufman, 2016). Esta sobrecarga desencadeia diversas reações inflamatórias locais e sistêmicas, contribuindo para a necrose do órgão e caracterizando-a como a $2^{a}$ causa mais frequente de indicação de transplante nos EUA por cirrose hepática (Wong et al., 2015).

Por sua etiologia multifatorial, envolvendo desde alimentação até condutas terapêuticas de outras comorbidades, a EHNA pode acometer crianças, jovens, adultos e idosos, com predominância após a quarta década de vida (Stal, 2015). Geralmente, apresenta-se com um quadro clínico silencioso nas fases mais agudas, desenvolvendo manifestações sistêmicas nos estágios mais avançados, nos quais a homeostase corporal não está sendo mantida mesmo com as reações compensatórias. Em razão disso, a suspeita e diagnóstico da EHNA está quase sempre relacionada à alteração das transaminases hepáticas ou por exames de imagens em consultas de rotina (European Association for the Studyof The Liver, \&European Association for the Study of Diabetes, 2016; European Association for Study of Liver, 2015).

O diagnóstico padrão-ouro da EHNA é feito através da análise histopatológica colhida por biópsia hepática. Entretanto, nem sempre é o método de escolha na prática clínica, devido ao seu caráter invasivo. Assim sendo, os exames laboratoriais e os de imagem tornam-se uma grande ferramenta no cotidiano. Destes, a ressonância magnética é o de escolha, porém, por ser pouco acessível, a ecografia abdominal é a mais solicitada. Alguns escores de avaliação podem ser analisados, como o NAFLD activity score (NAS) ou o steatosis, activity and fibrosis score (SAF) (European Association for the Study of 
The Liver, \& European Association for the Study of Diabetes, 2016).

Por fim, o tratamento é feito pela compensação das comorbidades associadas e controle dos fatores de risco, associados a uma mudança do estilo de vida com dieta balanceada e a prática de exercícios físicos rotineiramente, visando a perda de peso e o enquadramento no IMC adequado (Armstrong et al., 2016; Lassailly et al., 2015; Vilar et al., 2015). A terapia não medicamentosa foi demonstrada como a única eficaz em reverter o quadro da EHNA. Contudo, devido ao perfil clínico dos pacientes com essa comorbidade, apresenta-se com uma grande taxa de insucesso terapêutico, necessitando de uma terapia medicamentosa de suporte que, atualmente, não está totalmente elucidada (Arrese, Cabrera \& Barrera, 2015; Neuschwander et al., 2015). Esta é composta por medicamentos antidiabéticos, como a liraglutida e pioglitazona; insulinoterapia, vitamina E, dentre outros (Britton, Subramaniam \& Crawford, 2016; Lainé et al., 2017; Rametta et al., 2016).

Assim, o presente estudo propõe avaliar a eficácia do uso de liraglutida na EHNA, demostrando suas vantagens e desvantagens no tratamento clínico dos pacientes, além de orientar e informar os profissionais da área sobre qual a melhor opção no tratamento individualizado desta comorbidade.

\section{Metodologia}

Trata-se de um estudo qualitativo baseado nos preceitos de uma revisão sistemática de tratamento da literatura, com a finalidade de avaliar a utilização da Liraglutida na abordagem terapêutica medicamentosa na Esteatose Hepática Não Alcoólica (EHNA). O estudo qualitativo possibilita abordagens diferenciadas de uma mesma temática a partir da interpretação de cada autor, sendo responsável por formular diversos desfechos de um mesmo ponto de partida (Tuzzo \& Braga, 2016).

Para isto, foram utilizadas as bibliotecas virtuais US National Library of Medicine National Institutes of Health (PubMed), Biblioteca Virtual de Saúde (BVS), Scientific Electronic Library Online (SCIELO) e Literatura Latino-americana e do Caribe em Ciências da Saúde (LILACS), com o auxílio dos descritores em ciência da saúde (DeCS) para a consulta das palavras-chave: Non-alcoholic Fatty Liver Disease, Drug therapye, Liraglutide. Além disso, foram utilizados como critérios de inclusão: artigos dos últimos cinco anos, em humanos, sem restrição quanto ao sexo, etnia e idade, bem como, ensaios clínicos de ambas as fases e controlado.

Com as palavras-chaves, foram encontrados 132 artigos, sendo 67 no PubMed, 64 na BVS e 1 no LILACS. Quando restrito aos ensaios clínicos, essa proporção fica de 10 e 16, respectivamente, não sendo encontrado no LILACS. Após aplicação dos critérios de inclusão, o PubMed permanece com os 10 artigos; entretanto a BVS reduz para 12 e depois 10, respectivamente. Dessa forma, ficaram 20 artigos de mostra para a revisão sistemática, sendo 7 repetidos e 2 sem abranger o tema, resultando em 11 artigos finais. Esses dados foram representados na figura 1.

Em relação à qualidade das evidências, a classificação foi feita baseada nos critérios do Grading Quality of Evidence and Strengh of Recommendation (GRADE). Dos artigos, 1 ficou com a pontuação máxima de $+4,5$ receberam +3 devido ao viés de publicação e limitação metodológica. Dos quatro artigos restantes, dois receberam +2 e dois +1 devido à imprecisão e ao viés de publicação. 
Figura 1: Fluxograma dos ensaios clínicos encontrados nas bases de dados.

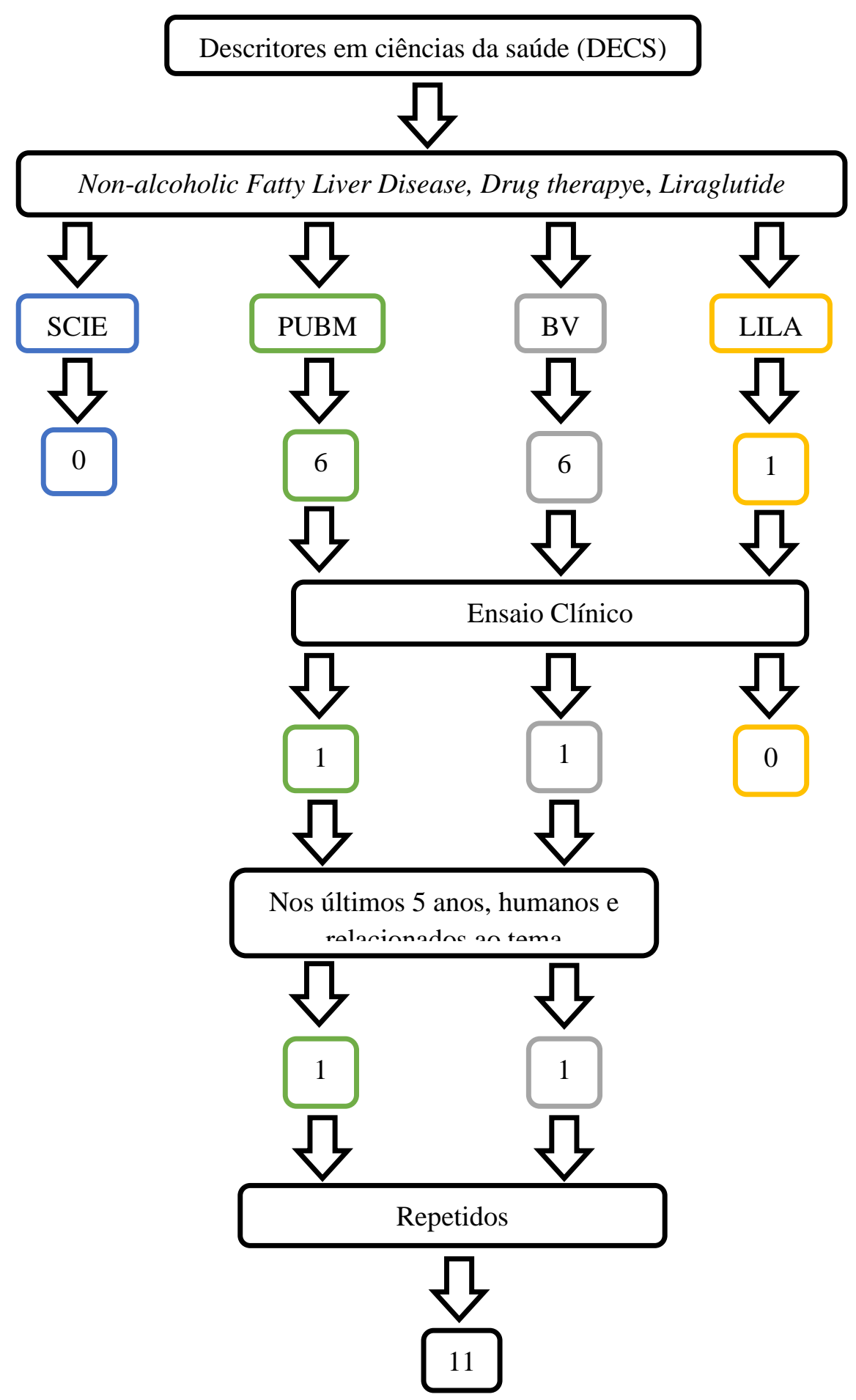

Fonte: Autores (2021).

Na Figura 1, observa-se que ocorre a concentração dos ensaios clínicos nas bases Pubmed e Bvs, e que nos últimos cinco anos congrega-se mais da metade dos ensaios publicados sobre o tema. 


\section{Resultados}

De acordo com a Tabela 1, é possível observar a distribuição dos ensaios clínicos em relação ao ano de publicação, tipo de estudo, base de dados encontrada e o país. Pela distribuição continental, 45,46\% dos artigos foram publicados na Ásia, seguidos pera Europa com 36,36\% e, por último, a América do Norte com 18,18\%. Além disso, 90,9\% dos ensaios clínicos foram encontrados nas Publicações Médicas (PUBMED) e 9,1\% na Biblioteca Virtual de Saúde (BVS).

Tabela 1: Especificação dos ensaios clínicos de acordo com o ano, base de dados e país.

\begin{tabular}{cccc}
\hline Autor & Tipo de estudo & Base de dados & País \\
\hline Armstronget al. (2016) & $\begin{array}{c}\text { Ensaio clínico randomizado duplo-cego } \\
\text { e controlado por placebo }\end{array}$ & PUBMED & Inglaterra \\
\hline Fenget al. (2018) & Ensaio clínico randomizado & PUBMED & China \\
Yanet al.(2019) & Ensaio clínico multicêntrico & PUBMED & China \\
Fenget al.(2017) & Ensaio clínico randomizado & PUBMED & China \\
\hline Armstrong et al.(2016) & $\begin{array}{c}\text { Ensaio clínico randomizado duplo-cego } \\
\text { e controlado por placebo }\end{array}$ & PUBMED & Inglaterra \\
Khooet al.(2017) & Ensaio clínico randomizado piloto & PUBMED & Singapura \\
Matikainenet al.(2018) & Ensaio clínico randomizado & PUBMED & Finlândia \\
\hline Bouchiet al.(2017) & Ensaio clínico randomizado & PUBMED & Japão \\
\hline Smits et al.(2016) & Ensaio clínico randomizado controlado \\
por placebo & PUBMED & Holanda \\
\hline Gawrieh, & Ensaio clínico randomizado & PUBMED & Estados \\
Chalasani(2018) & Unidos
\end{tabular}

Fonte: Autores (2021).

Após a análise dos estudos, suas classificações quanto ao nível de evidência foram seguidas pelos critérios de Grading quality of evidence and strengh of recommendation (GRADE). Os fatores de diminuição do nível de evidência abrangem as limitações metodológicas, presença de inconsistências nos resultados, imprecisão das informações e ser oriundo de uma evidência indireta. Na tabela 2, os ensaios clínicos estão discriminados quanto a sua qualidade e índice de GRADE. Salienta-se que uma alta qualidade, presente em $54,55 \%$ dos estudos, confere uma forte confiança nos resultados apresentados, e a média qualidade outorga uma confiança moderada, mas utilizável como dado científico, e foi presente em $45,45 \%$ dos estudos. 
Tabela 2: Classificação da qualidade dos estudos segundo os critérios GRADE: Fatores responsáveis pela diminuiçãodo nível de evidência.

\begin{tabular}{|c|c|c|c|c|c|c|}
\hline Autor & $\begin{array}{c}\text { Limitações } \\
\text { metodológica }\end{array}$ & Inconsistência & Evidência indireta & Imprecisão & Qualidade & $\begin{array}{c}\text { Índice de } \\
\text { Grade }\end{array}$ \\
\hline $\begin{array}{c}\text { Armstronget al. } \\
\text { (2016) }\end{array}$ & Não há & Não há & Não há & Não há & Alta & +4 \\
\hline Fenget al. (2018) & Presente & Não há & Não há & Não há & Alta & +3 \\
\hline Yanet al.(2019) & Presente & Não há & Não há & Não há & Alta & +3 \\
\hline Fenget al.(2017) & Presente & Não há & Não há & Não há & Alta & +3 \\
\hline $\begin{array}{l}\text { Armstrong et } \\
\text { al.(2016) }\end{array}$ & Presente & Não há & Não há & Não há & Alta & +3 \\
\hline Khooet al.(2017) & Presente & Não há & Não há & Presente & Média & +1 \\
\hline $\begin{array}{c}\text { Matikainenet } \\
\text { al.(2018) }\end{array}$ & Presente & Não há & Não há & Não há & Média & +2 \\
\hline Bouchiet al.(2017) & Presente & Não há & Não há & Não há & Média & +2 \\
\hline Smits et al.(2016) & Presente & Presente & Não há & Não há & Média & +1 \\
\hline $\begin{array}{c}\text { Gawrieh, } \\
\text { Chalasani(2018) }\end{array}$ & Presente & Não há & Não há & Não há & Alta & +3 \\
\hline Caldwell(2017) & Presente & Presente & Presente & Não há & Média & +1 \\
\hline
\end{tabular}

Fonte: Autores (2021).

O tratamento da esteatose hepática não-alcoólica (EHNA) é constituído por uma base não medicamentosa, que abrange dieta e a prática de exercícios físicos, e uma medicamentosa que nesse estudo será representado pela liraglutida, um análogo do hormônio peptídeo semelhante a glucagon 1 (GLP-1). O considerado padrão-ouro, atualmente, é o eixo não medicamentoso (SMITS et al., 2016).

Os estudos foram discriminados quanto à posologia da liraglutida utilizada, duração do tratamento e exame utilizado para diagnóstico da EHNA, uma vez que são imprescindíveis para o resultado final obtido. Segundo a tabela 3, 81,81\% dos ensaios utilizaram a liraglutida em uma dose de 1,8mg diários. Em relação à duração do tratamento, cerca de 27,27\% duraram 48 semanas, seguido de 12, 24 e 26 semanas com 18,18\% cada. Por fim, o exame mais utilizado para o diagnóstico da EHNA foi a biópsia hepática (padrão-ouro), com 36,36\%, seguido pela ultrassonografia hepática (USG) e pela espectroscopia de próton por ressonância magnética (H1-ERM) com $18,18 \%$ cada. 
Tabela 3: Detalhamento do ensaio clínico quanto a posologia utilizada, duração do tratamento e exame utilizado no diagnóstico da EHNA.

\begin{tabular}{cccc}
\hline Autor & Posologia & $\begin{array}{c}\text { Duração da terapia } \\
\text { (semanas) }\end{array}$ & $\begin{array}{c}\text { Exame } \\
\text { utilizado para } \\
\text { o diagnóstico }\end{array}$ \\
\hline Armstronget al. (2016) & $1,8 \mathrm{mg}$ & 48 & Biópsia \\
\hline Fenget al. (2018) & $1,8 \mathrm{mg}$ & 24 & USG \\
\hline Yanet al.(2019) & $1,8 \mathrm{mg}$ & 26 & USG \\
\hline Fenget al.(2017) & $1,8 \mathrm{mg}$ & 24 & Biópsia \\
\hline Armstrong et al.(2016) & $1,8 \mathrm{mg}$ & 26 & RM \\
\hline Khooet al.(2017) & $3,0 \mathrm{mg}$ & 12 & TC \\
\hline Matikainenet al.(2018) & $1,8 \mathrm{mg}$ & 36 & Hiópsia \\
\hline Bouchiet al.(2017) & $0,9 \mathrm{mg}$ & 12 & Biópsia \\
\hline Smits et al.(2016) & $1,8 \mathrm{mg}$ & 48 & \\
\hline Gawrieh, & $1,8 \mathrm{mg}$ & 48 & \\
\hline Chalasani(2018) & $1,8 \mathrm{mg}$ & & \\
\hline
\end{tabular}

Biópsia: padrão-ouro; USG: ultrassonagrafia; MRI-PDFF: magnetic resonance imaging protondensity fat fraction; RM: ressonância magnètica; H1-ERM: espectroscopia de próton por ressonância magnética; TC: tomografia computadorizada;

Fonte: Autores (2021).

Segundo Armstrong et al., 2016, 39\% dos pacientes com EHNA tratados com liraglutida evoluíram para a resolução definitiva desta, ou seja, houve o desaparecimento do balonamento nos hepatócitos sem agravamento da fibrose hepática, confirmada pela biópsia comparativa no período inicial e após as 48 semanas de tratamento, assim como foi elucidado no Gráfico 1. Em contrapartida, essa melhora só foi observada em 2\% dos pacientes do grupo placebo ao final das 48 semanas. 
Gráfico 1: Evolução da EHNA após 48 semanas de terapia.

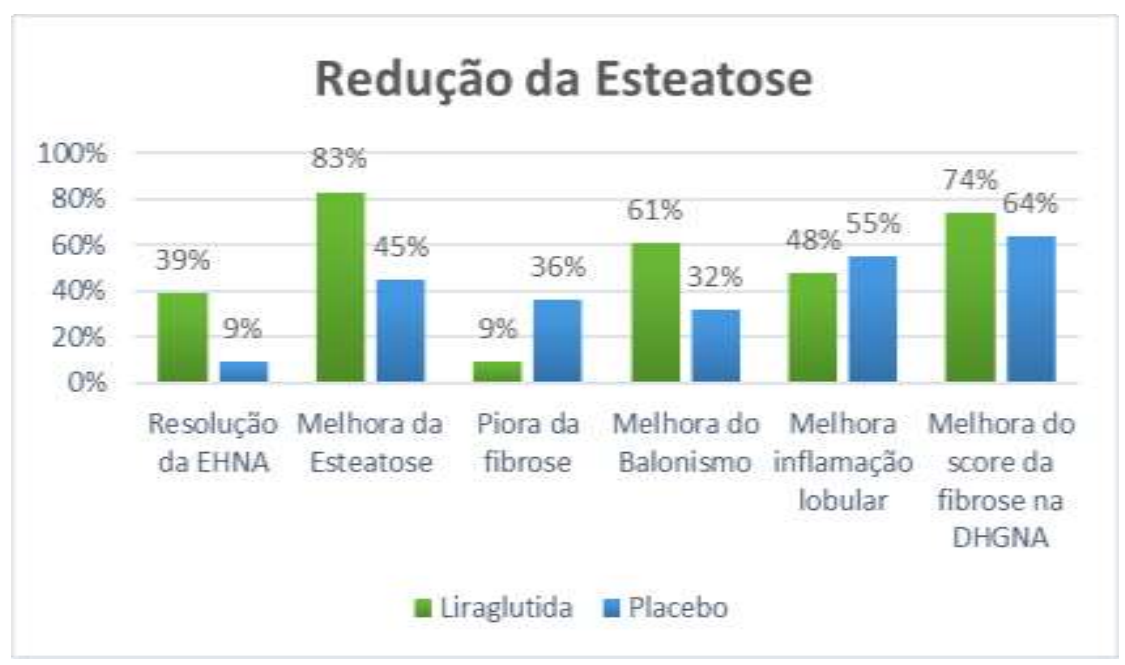

Fonte: Adaptado do Gawrieh \& Chalasani (2018).

O análogo do hormônio GLP-1 atua em várias frentes na EHNA. A mais conhecida seria sua diminuição do peso, acima de 5\%, devido à supressão do apetite e alteração dos movimentos peristálticos, se assemelhando à mudança do estilo de vida (Feng et al., 2017; Khoo et al., 2017). Entretanto, os estudos demostraram que as outras vias são mais eficazes, como a diminuição da secreção de glucagon, o aumento da liberação de insulina, a supressão da lipogênese de novo (DNL), o aumento da oxidação dos ácidos graxos, promoção da autofagia e o retardamento do esvaziamento gástrico (Gawrieh \& Chalasani, 2018).

No grupo liraglutida, ainda é possível observar uma diminuição nas concentrações da gama glutamiltransferase (Gama GT), da alanina aminotransferase (ALT), do aspartatoaminotranferase (AST) e dos biomarcadores séricos de lesão do hepatócito (caspase e citoqueratina 18 clivada) após a finalização dos estudos. Além disso, associou-se a uma redução ponderal, do Índice de Massa Corporal (IMC)e das concentrações de hemoglobina glicada (HbA1c) em comparação ao placebo, logo nas primeiras 12 semanas. Entretanto, após 12 semanas de finalização dos estudos, é observado a retomada dos valores metabólicos e ponderais basais após a descontinuação da medicação (Armstrong et al., 2016; Feng et al., 2017; Feng et al., 2018; Gawrieh \& Chalasani, 2018; Khoo et al., 2017).

Segundo Feng et al., 2018, a perda ponderal foi mais evidente no tronco, membros, no ginecoide e androide. Observou-se uma diminuição significativa na circunferência abdominal (CA), sendo constatado a perda tanto de massa gorda como massa magra, preferencialmente da primeira. Em relação ao controle glicêmico, foi acompanhado pelo teste de padrão de tolerância à glicose (TOTG) após 24 semanas nos tempos 0, 30, 60 e 120 min e pela Hemoglobina glicada (HbA1c), onde a liraglutida alcançou o maior controle em relação às outras medicações (metformina e glicazida), diminuindo a HbA1c igual ou superior a 2,5\% do valor inicial, assim como foi mostrado nos gráficos 2 e 3(Feng et al., 2017; Feng et al., 2018). 
Gráfico 2: Redução do peso corporal.

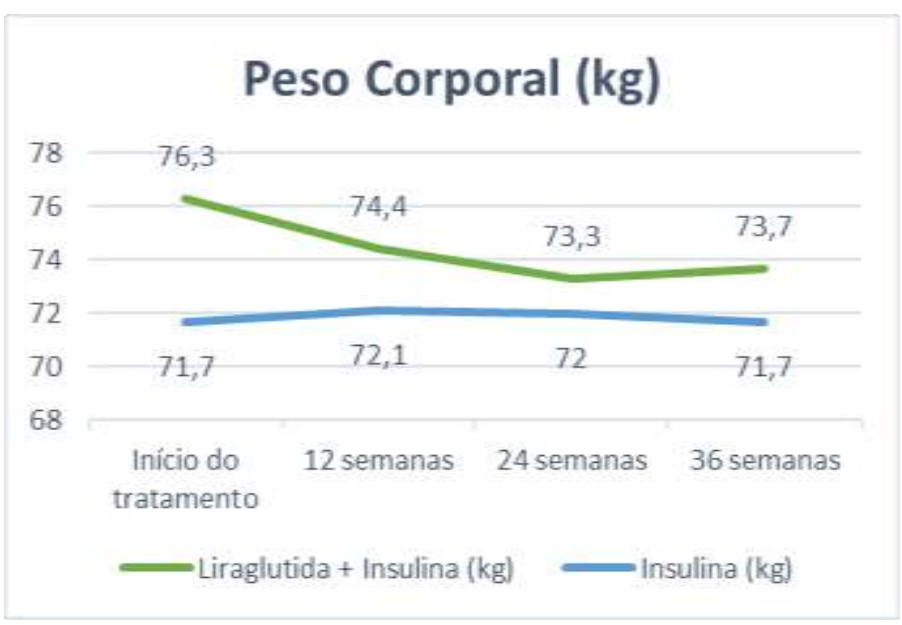

Fonte: Autores (2021).

Adaptado Bouchi et al., (2017).
Gráfico 3: Níveis de HbA1c durante a terapia.

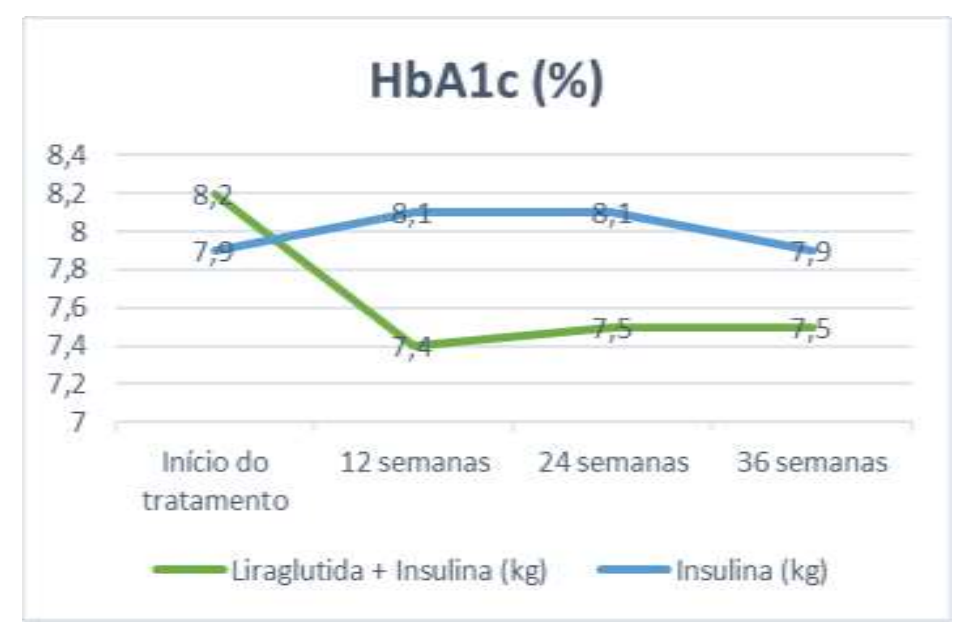

Fonte: Autores (2021).

Adaptado Bouchi et al., (2017).

Após as 48 semanas, segundo Feng et al., 2017, houve redução da gordura intra-hepática (IHF), por esta ser associada à glicotoxicidade e aos altos índices de HbA1c, AST e ALT. No mais, melhorou e restaurou a sensibilidade hepática e global à insulina, reduziu o DNL, os metabólitos lipotóxicos, mediadores pró-inflamatórios, a ação lipogênica e os níveis de leptina e adiponectina (Armstrong et al., 2016). A função cardioprotetora reduziu a incidência da arteriosclerose, diminuindo o risco de Acidente Vascular Cerebral (AVC) e Infarto Agudo do Miocárdio (IAM) (Bouchi et al., 2017).

Todavia, foi relacionado a efeitos adversos de leve a moderada gravidade, principalmente pelos efeitos gastrointestinais, tais como náuseas, vômitos, supressão do apetite, diarreia, distensão abdominal, constipação e reações locais pela injeção, apresentados a seguir no Gráfico 4 (Armstrong et al., 2016; Caldwell, 2017; Feng et al., 2017; Feng et al., 2018; Gawrieh \& Chalasani, 2018).

Gráfico 4: Relação dos principais efeitos colaterais com o uso da liraglutida versus placebo.

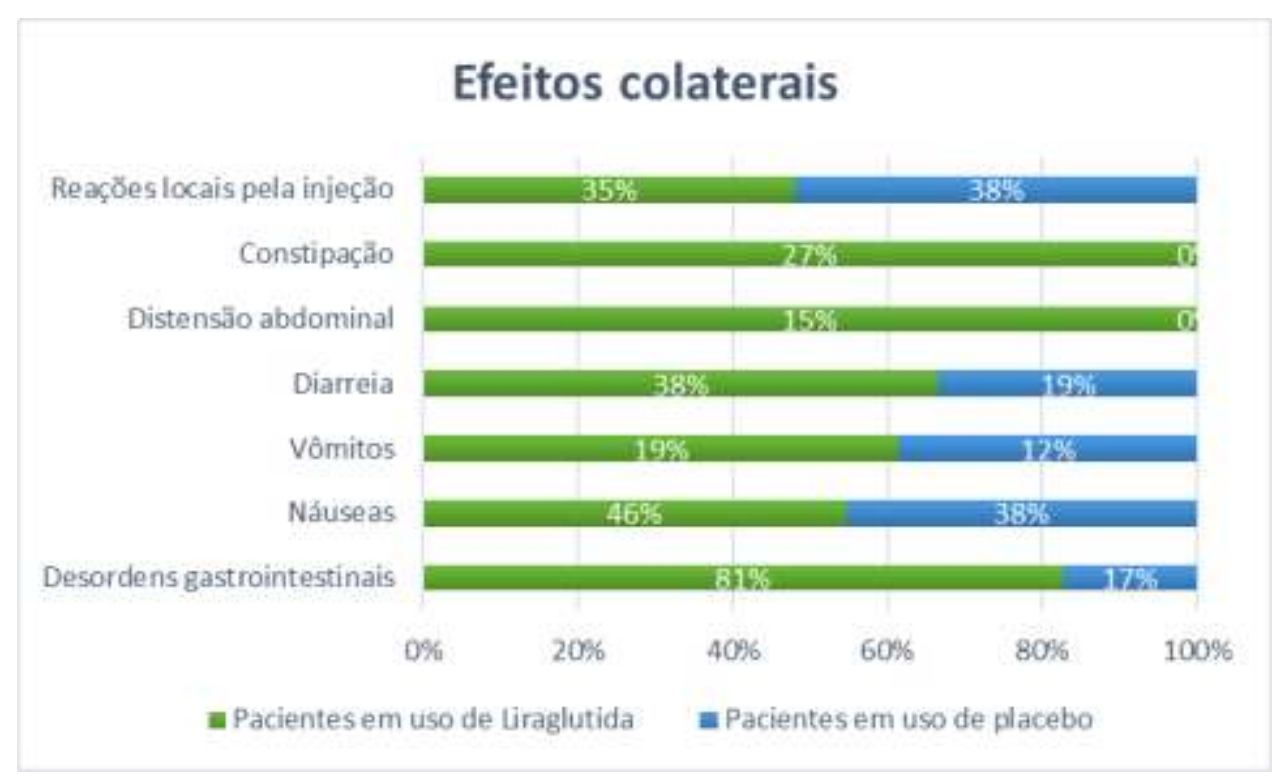

Fonte: Autores (2021). Adaptado Armstrong et al. (2016). 


\section{Discussão}

Atualmente, o protocolo inicial para o tratamento da esteatose hepática não-alcoólica (EHNA) é uma associação entre mudanças no padrão alimentar e atividade física. Entretanto, a baixa disponibilidade de tempo, o ambiente obesogênico, o sedentarismo e a diminuição do gasto energético encontrados na sociedade contemporânea vão de encontro a essa opção terapêutica nas condutas médicas (Khoo et al., 2017; Smits et al., 2016).

É evidente que as alterações metabólicas patológicas atuais não advêm de um único fator causal, e sim de uma associação e potencialização destes no decorrer do tempo. Na EHNA não seria diferente. Ela surge inicialmente com uma resistência insulínica correlacionada com um aumento da lipogênese global, aumentando as concentrações corporais de ácidos graxos não esterificados e de metabólitos tóxicos derivados da degradação dos triglicerídeos (TG), formando os insultos lipotóxicos primários que contribuem para o aumento do risco cardiovascular e um meio pró-inflamatório (Armstrong et al., 2016).

O quadro clínico é silencioso, e sua observação e diagnóstico são feitos, geralmente, na presença de suas complicações, como as varizes esofágicas e ascite. Possui uma prevalência global de 30\% na população, e nos pacientes com diabetes mellitus tipo 2 (DM2) sobe para 70\%, devido à resistência insulínica. A EHNA necessita de uma atenção redobrada por ser uma lesão pré-neoplásica, uma vez que evolui para cirrose hepática, e deste para carcinoma hepatocelular (Caldwell et al., 2017; Smits et al., 2016).

Diante disso, uma terapia medicamentosa que tenha a mesma eficácia da conduta não medicamentosa, e ainda não dependa inteiramente da ação do paciente é a mais eficaz nos dias atuais. Nesse contexto, temos a liraglutida, um análogo sintético da incretina GLP-1, que atua diretamente na resistência insulínica global e no fígado, além de reduzir a exposição hepática aos metabólitos tóxicos (Feng et al., 2017; Khoo et al., 2017).

Segundo os ensaios clínicos, o tratamento com $3 \mathrm{mg}$ de liraglutida e duração de 48 semanas obtém uma desaceleração e melhora da EHNA, além de retardar a progressão da fibrose e diminuir a inflamação hepática em pacientes com e sem DM2 associado, através de bastantes frentes que visam a homeostase corporal (Caldwell et al., 2017; Gawrieh \& Chalasani, 2018; Smitset al., 2016).Tratamentos com duração de 26 e 36 semanas também obtiveram sucesso na resolutividade da EHNA (Bouchi et al., 2017; Khoo et al., 2017). Entretanto, terapias com duração iguais ou menores a 24 semanas não obtiveram resolutividade total na EHNA, principalmente menores que 12 semanas, onde não se observou nenhuma alteração na gordura intra-hepática (Matikainem et al., 2018; Smits et al., 2016).

A perda ponderal entre 5\%a $10 \%$ obtida através da supressão do apetite no eixo cerebral, por ser agonista de um receptor intestinal que proporciona saciedade, retardamento do esvaziamento gástrico e alterações nos movimentos peristálticos, que diminuem a absorção dos carboidratos, resultando na diminuição do IMC e da CA, consequentemente. Além disso, promove uma diminuição da liberação do glucagon, um hormônio que é diabetogênico; em contrapartida, aumenta os níveis de insulina, melhorando os índices glicêmicos do paciente e restaurando a sensibilidade hepática e global para a mesma (Armstrong et al., 2016; Feng et al., 2017; Feng et al., 2018; Yan et al., 2019).

O estado hiperglicêmico é nocivo devido ao seu estímulo pró-inflamatório e lipogênese de novo (DNL). É constatado através da glicemia de jejum (GJ) e da HbA1c. A DNL é uma via adicional na lipogênese bem presente no tecido hepático, responsável por uma maior formação de triglicerídeos, ácidos graxos e glicerol, os quais participam diretamente da patogênese da EHNA. A liraglutida suprime a via da DNL, pois diminui o estado hiperglicêmico, e com isso diminui a ativação da proteína ligadora do elemento de responsividade a carboidratos. Esse seria o estímulo necessário para que ocorra a DNL. Suprimindo e inativando o início da via lipogênica, consequentemente os níveis dos seus produtos também reduzirá, como é o caso dos triglicerídeos, ácidos graxos e glicerol (Armstrong et al., 2016; Matikainem et al., 2018). 
Em relação ao fígado, é observado uma melhora das enzimas hepáticas tais como gama GT, AST e ALT, além dos biomarcadores séricos (caspase e citoqueratina 18 clivada). Outros achados são a normalização dos níveis de leptina e adiponectina, e um efeito cardioprotetor por diminuição da arteriosclerose induzida pelo controle do perfil lipídico, diminuindo o risco de AVC e IAM (Bouchi et al., 2017; Gawrieh \& Chalasani, 2018; Khoo et al., 2017).

Apesar da sua grande eficácia frente à EHNA, a liraglutida apresenta efeitos colaterais, principalmente, relacionados ao trato gastrointestinal, como náuseas, vômitos, constipação, diarreia, distensão abdominal entre outros, os quais, segundo os estudos, não foram motivos para o abandono da terapia (Armstrong et al., 2016; Caldwell, 2017; Feng et al., 2017; Feng et al., 2018; Gawrieh \& Chalasani, 2018; Yan et al., 2019).

\section{Conclusão}

A liraglutida foi resolutiva na EHNA, tanto na sua estabilidade como regressão. Além disso, contribuiu para a estabilização da fibrose hepática e melhora da inflamação dos hepatócitos. Vale salientar que na sua terapia é necessário a avaliação de duas variáveis: a duração do tratamento e a posologia utilizada. A primeira variável interferiu positivamente na reparabilidade da EHNA após 26 semanas. Uma duração mais curta, principalmente menor que 12 semanas não influenciou melhores resultados. Em relação à segunda variável, foi alcançado o resultado almejado nas concentrações de $0,9 \mathrm{mg}, 1,8 \mathrm{mg} \mathrm{e}$ 3,0 mg. Entretanto, doses maiores foram relacionadas à duração de tratamento menor. A dose de 1,8 mg durante 48 semanas foi considerada a melhor conduta terapêutica estabelecida pelos estudos.

Somando-se a isso, são necessários mais ensaios clínicos para a descoberta de mais benefícios da medicação.

\section{Referências}

Araújo, A. R. et al. (2018). Global epidemiology of non-alcoholic fatty liver disease/non-alcoholic steatohepatitis: What we need in the future. Liver International, 38, 47-51.

Armstrong, M. J. et al. (2016). Glucagon-like peptide 1 decreases lipotoxicity in non-alcoholic steatohepatitis. Journal of hepatology, 64(2), 399-408.

Armstrong, M. J. et al. (2016). Liraglutide safety and efficacy in patients with non-alcoholic steatohepatitis (LEAN): a multicentre, double-blind, randomised, placebo-controlled phase 2 study. The Lancet, 387(10019), 679-690.

Arrese, M., Cabrera, D., \& Barrera, F. (2015). Obeticholic acid: expanding the therapeutic landscape of NASH. Annals of hepatology, 14(3), 430-432.

Bouchi, R. et al (2016). Reduction of visceral fat by liraglutide is associated with ameliorations of hepatic steatosis, albuminuria, and micro-inflammation in type 2 diabetic patients with insulin treatment: a randomized control trial. Endocrine journal, EJ16-0449.

Britton, L. J., Subramaniam, V. N., \& Crawford, D. H. (2016). Iron and non-alcoholic fatty liver disease. World journal of gastroenterology, $22(36), 8112$.

Caldwell, S. (2016). NASH Therapy: Omega 3, Vitamin E, Insulin Sensitizers and Statins. In Single Topic Symposium (STS) (Vol. 2016, No. 2, pp. 25-26).

European Association for the Study of The Liver, \& European Association for the Study of Diabetes (EASD. (2016). EASL-EASD-EASO Clinical Practice Guidelines for the management of non-alcoholic fatty liver disease. Obesity facts, 9(2), 65-90.

Estes, C. et al. (2018). Modeling the epidemic of nonalcoholic fatty liver disease demonstrates an exponential increase in burden of disease. Hepatology, 67(1), $123-133$.

European Association for Study of Liver. (2015). EASL-ALEH Clinical Practice Guidelines: Non-invasive tests for evaluation of liver disease severity and prognosis. Journal of hepatology, 63(1), 237-264.

Feng, W. et al. (2017). Randomized trial comparing the effects of gliclazide, liraglutide, and metformin on diabetes with non-alcoholic fatty liver disease. Journal of diabetes, $9(8), 800-809$.

Feng, W. et al. (2019). Effects of liraglutide, metformin and gliclazide on body composition in patients with both type 2 diabetes and non-alcoholic fatty liver disease: a randomized trial. Journal of diabetes investigation, 10(2), 399-407.

Gawrieh, S., \& Chalasani, N. (2018). Emerging treatments for nonalcoholic fatty liver disease and nonalcoholic steatohepatitis. Clinics in liver disease, 22(1), 189-199.

Khoo, J. et al. (2017). Comparative effects of liraglutide $3 \mathrm{mg}$ vs structured lifestyle modification on body weight, liver fat and liver function in obese patients with non-alcoholic fatty liver disease: a pilot randomized trial. Diabetes, Obesity and Metabolism, 19(12), 1814-1817. 
Kirpich, I. A., Marsano, L. S., \& McClain, C. J. (2015). Gut-liver axis, nutrition, and non-alcoholic fatty liver disease. Clinical biochemistry, 48(13-14), 923930.

Lainé, F. et al. (2017). Metabolic and hepatic effects of bloodletting in dysmetabolic iron overload syndrome: A randomized controlled study in 274 patients. Hepatology, 65(2), 465-474.

Lassailly, G. et al. (2015). Bariatric surgery reduces features of nonalcoholic steatohepatitis in morbidly obese patients. Gastroenterology, 149(2), 379-388.

Lebeaupin, C. et al. (2018). Endoplasmic reticulum stress signalling and the pathogenesis of non-alcoholic fatty liver disease. Journal of hepatology, 69(4), 927-947.

Lonardo, A. et al. (2015). Epidemiological modifiers of non-alcoholic fatty liver disease: Focus on high-risk groups. Digestive and Liver Disease, 47(12), 9971006 .

Matikainen, N. et al. (2019). Liraglutide treatment improves postprandial lipid metabolism and cardiometabolic risk factors in humans with adequately controlled type 2 diabetes: A single-centre randomized controlled study. Diabetes, obesity and metabolism, 21(1), 84-94.

Monaco-Ferreira, D. V., \& Leandro-Merhi, V. A. (2017). Weight regain 10 years after Roux-en-Y gastric bypass. Obesity surgery, 27(5), 1137-1144.

Neuschwander-Tetri, B. A. et al. (2015). Farnesoid X nuclear receptor ligand obeticholic acid for non-cirrhotic, non-alcoholic steatohepatitis (FLINT): a multicentre, randomised, placebo-controlled trial. The Lancet, 385(9972), 956-965.

Petit, J. M. et al. (2017). Effect of liraglutide therapy on liver fat content in patients with inadequately controlled type 2 diabetes: the Lira-NAFLD study. The Journal of Clinical Endocrinology \& Metabolism, 102(2), 407-415.

Rametta, R. et al. (2016). Hepcidin resistance in dysmetabolic iron overload. Liver International, 36(10), $1540-1548$.

Rinella, M. E. (2015). Nonalcoholic fatty liver disease: a systematic review. Jama, 313(22), 2263-2273.

Rotman, Y., \& Sanyal, A. J. (2017). Current and upcoming pharmacotherapy for non-alcoholic fatty liver disease. Gut, 66(1), 180-190.

Singh, S. et al. (2015). Fibrosis progression in nonalcoholic fatty liver vs nonalcoholic steatohepatitis: a systematic review and meta-analysis of paired-biopsy studies. Clinical gastroenterology and hepatology, 13(4), 643-654.

Smits, M. M. et al. (2016). Twelve week liraglutide or sitagliptin does not affect hepatic fat in type 2 diabetes: a randomised placebo-controlled trial. Diabetologia, 59(12), 2588-2593.

Stål, P. (2015). Liver fibrosis in non-alcoholic fatty liver disease-diagnostic challenge with prognostic significance. World Journal of Gastroenterology: WJG, 21(39), 11077.

Tuzzo, S. A., \& Braga C. F. (2016). O processo de triangulação da pesquisa qualitativa: o metafenômeno como gênese. Revista Pesquisa Qualitativa, São Paulo, SP, v.4, n.5, p. 140-158.

Van Herck, M. A., Vonghia, L., \& Francque, S. M. (2017). Animal models of nonalcoholic fatty liver disease—a starter's guide. Nutrients, 9(10), 1072.

Vilar-Gomez, E. et al. (2015). Weight loss through lifestyle modification significantly reduces features of nonalcoholic steatohepatitis. Gastroenterology, 149(2), 367-378.

Wang, M., \& Kaufman, R. J. (2016). Protein misfolding in the endoplasmic reticulum as a conduit to human disease. Nature, 529(7586), 326-335.

Willy, J. A. et al. (2015). CHOP links endoplasmic reticulum stress to NF- $\mathrm{kB}$ activation in the pathogenesis of nonalcoholic steatohepatitis. Molecular biology of the cell, 26(12), 2190-2204.

Wong, R. J. et al. (2015). Nonalcoholic steatohepatitis is the second leading etiology of liver disease among adults awaiting liver transplantation in the United States. Gastroenterology, 148(3), 547-555.

Yan, J. et al. (2019). Liraglutide, sitagliptin, and insulin glargine added to metformin: the effect on body weight and intrahepatic lipid in patients with type 2 diabetes mellitus and nonalcoholic fatty liver disease. Hepatology, 69(6), 2414-2426.

Younossi, Z. M. et al. (2016). Global epidemiology of nonalcoholic fatty liver disease-meta-analytic assessment of prevalence, incidence, and outcomes. Hepatology, 64(1), 73-84.

Zelber-Sagi, S., Godos, J., \& Salomone, F. (2016). Lifestyle changes for the treatment of nonalcoholic fatty liver disease: a review of observational studies and intervention trials. Therapeutic advances in gastroenterology, 9(3), 392-407. 